\title{
Diseño de infraestructura vial con pavimento flexible para mejora de transitabilidad de la avenida Industrial, Lurín, Lima
}

\author{
Design of road infrastructure with flexible pavement to improve the \\ traffic of Avenida Industrial, Lurín, Lima
}

\author{
Sleyther Arturo De La Cruz Vega ${ }^{1}$, Guirlo Alejandro Paredes Cahuana ${ }^{2}$
}

Recibido: Noviembre 2021

Aceptado: Noviembre 2021

\begin{abstract}
Resumen.- El objetivo de la investigación es determinar el diseño de pavimento flexible para la mejora de la transitabilidad vehicular. La metodología es de tipo aplicada, diseño no experimental y de enfoque cuantitativo. El universo poblacional está conformado por toda la avenida Industrial del distrito de Lurín. La muestra correspondiente a 2 kilómetros y el muestreo es no probabilístico. Los resultados del estudio muestran que la estructura del pavimento es $7.5 \mathrm{~cm}$ de carpeta asfáltica, $20 \mathrm{~cm}$ de base y $15 \mathrm{~cm}$ de subbase. Las conclusiones indican que el diseño del pavimento flexible servirá para mejorar la transitabilidad vehicular de la avenida Industrial, Lurín, Lima. Según estudio de capacidad de carga realizado en 6 calicatas dio un promedio de CBR 32.50. El estudio vehicular realizado durante 7 días para el diseño de pavimento flexible nos dio un ESAL de 1.22 E+07 realizado para un periodo de 20 años. Esta investigación presenta un diseño acorde con la cantidad de vehículos que se desplazan por dicho lugar, debido a que el conteo vehicular se realizó de manera minuciosa utilizando los periodos establecidos en el reglamento del MTC.
\end{abstract}

Palabras clave: pavimento, asfalto, transitabilidad, estudio, vehículo.

Summary.- The objective of the research is to determine the flexible pavement design for the improvement of vehicular traffic. The methodology is of an applied type, non-experimental design and a quantitative approach. The population universe is made up of the entire Industrial Avenue of the Lurin district. The sample corresponding to 2 kilometers and the sampling is nonprobabilistic. The results of the study show that the pavement structure is $7.5 \mathrm{~cm}$ of asphalt layer, $20 \mathrm{~cm}$ of base and $15 \mathrm{~cm}$ of subbase. The conclusions indicate that the design of the flexible pavement will serve to improve the vehicular traffic of Avenida Industrial, Lurín, Lima. According to a load capacity study carried out in 6 pits, it gave an average of CBR 32.50. The vehicular study carried out during 7 days for the flexible pavement design gave us an ESAL of 1.22 E + 07 carried out for a period of 20 years. This investigation presents a design in accordance with the number of vehicles that move through said place, because the vehicle count was carried out in a meticulous way using the periods established in the MTC regulations.

Keywords: pavement, asphalt, walkability, study, vehicle.

\footnotetext{
1 Facultad de Ingeniería y Arquitectura, Universidad Cesar Vallejo (Perú), sleyther@ucvvirtual.edu.pe, ORCID iD: https://orcid.org/0000-0003-0254-301X

2 Facultad de Ingeniería y Arquitectura, Universidad Cesar Vallejo (Perú), guirlo.paredes@ gmail.com, ORCID iD: https://orcid.org/0000-0001-8342-0163
} 
1. Introducción. - A nivel mundial la infraestructura vial es muy importante por ser el eje principal de la economía y desarrollo de un país, además de conectar las áreas de producción con las de consumo, es allí donde radica la importancia de que estas se encuentren en buen estado y se reduzcan los grandes costos de transporte por mala infraestructura vial. [1].

Las vías en el Perú todavía se encuentran en desarrollo debido a la falta de una adecuada gestión de procesos y planificación, sumando a esto la falta de inversión privada y el déficit en la infraestructura vial, generando restricciones para el desarrollo de la economía peruana [2].

En el distrito de Lurín específicamente en las zonas rurales y urbanas hay un gran déficit vial, a estas restricciones se suma la geografía de los terrenos el cual es muy agresiva, provocando la desarticulación de la ciudas [3].

El desarrollo económico de ciertos sectores industriales hace de gran importancia la conexión vial, ubicándose el nuevo motor industrial del país en Lurín. Pese al gran potencial económico de los últimos años, actualmente nuestra capital solo cuenta con 2,700 Ha destinadas a dicho sector. [4]. Un tramo de la avenida Industrial actualmente no se encuentra pavimentada, siendo esta la vía principal para grandes empresas y al no estar al $100 \%$ operativa afecta directamente al desarrollo económico del sector industrial, dificultando el desplazamiento de los pobladores y de los vehículos [5].

Según la explicación de la problemática se plantea el siguiente problema general ¿Cómo el diseño de pavimento flexible mejora la transitabilidad de la Avenida Industrial, distrito de Lurín, Lima?

La justificación económica está enfocada en el desarrollo monetario de la zona en estudio, debido a la gran cantidad de industrias ubicadas en el sector A, contribuyendo al pago de impuestos prediales y generando fuentes de trabajo para la población. Actualmente el sector industrial se está reactivando luego de pasar por un periodo de inestabilidad económica a causas del COVID-19 consolidando a Lurín en el foco de desarrollo de lima. [6].

La justificación ambiental está enfocada en la reducción del polvo generado y daños a la salud de las poblaciones aledañas de la zona de estudio.

La justificación metodológica permitirá mejorar los procedimientos del diseño basado en la metodología AASHTO y el análisis vehicular. La justificación por conveniencia esta enfocado por el beneficio a las empresas industriales colindantes con la avenida Industrial que están en vía de desarrollo, debido a la necesidad de nuevas vías por la cual se puedan trasladar los vehículos y sus productos [7].

El objetivo general de acuerdo con la problemática y las justificaciones del presente trabajo desarrollado es determinar el diseño de pavimento flexible para la mejora de la transitabilidad vehicular de la avenida Industrial, Lurín, Lima.

2. Marco teórico. - Los pavimentos están conformados por varias capas como la carpeta asfáltica, la base y la subbase que descansan sobre la subrasante o terreno natural, toda esta estructura trasmite su carga y distribuye sus esfuerzos al terreno de fundación originados por los vehículos [8].

El pavimento flexible que está estructurado por la capa de rodadura de material aglomerante, agregados, aditivos y capas granulares como la base y subbase. [9].

La metodología de diseño tiene parámetros establecidos como el cálculo de ESAL de diseño, el Módulo de Resiliencia, las condiciones climáticas y de drenaje.

Las fallas del pavimento son el exagerado incremento de la carga vehicular, mal proceso constructivo, falta de mantenimiento, factores climáticos, proyecto deficiente, fallas superficiales, fallas estructurales [10].

Algunos factores importantes para tener en cuenta al momento de diseñar la estructura del pavimento es la subrasante y el nivel en que se encuentra, ya que debe de estar por encima del nivel de la napa freática con un mínimo de $0.60 \mathrm{~m} \mathrm{[11].}$

El tránsito se calcula mediante mediciones diarias se recomienda por 7 días ya que los vehículos 
que circulan en la vía son de diferentes características, verificar la cantidad de ejes equivalentes de $8.2 \mathrm{t}$ a $13 \mathrm{t}$ que circulan en el carril [12].

El módulo de resiliente muestra los parámetros utilizados y los esfuerzos que representan las cargas repetidas proporcionadas al suelo [13]. Una vez construido el pavimento con el paso del tiempo se presenta algunos problemas o fallas las cuales ya se han estudiado a profundidad y sirve como base para las soluciones más idóneas a los problemas que se presentan a futuro [14].

Las metodologías de diseño de pavimento flexible son el método empírico, que está basado en el análisis mecanístico de cada capa del paquete estructural diseñado, el método de diseño analítico en el que cada capa del paquete estructural está sujeto a deformaciones y programas de elementos finitos para el diseño de pavimentos. [15].

El método AASHTO 1993 se aplica tanto para diseños de pavimentos flexibles y rígidos, utilizando un modelo de ecuación definido, que permite obtener los números estructurales que se emplean en la determinación de los espesores. [16].

3. Metodología. - La investigación es de tipo aplicada, porque busca adquirir nuevos conocimientos mediante la aplicación de teorías y a la vez mejora situaciones de la vida cotidiana [17].

El diseño de investigación es no experimental transeccional descriptiva porque describe y analiza los diversos componentes sin alterar la variable [18].

Según el análisis realizado es una investigación cuantitativa con criterios a utilizar como la recolección de datos durante la ejecución del diseño de la estructura del pavimento [19].

Su universo poblacional está conformado por toda la avenida industrial del distrito de Lurín, Lima. Para la investigación tiene como muestra cierto sector de la avenida industrial, comprendido entre las avenidas Gasoducto y la avenida Sumapacha, correspondientes a 2 kilómetros. [20].

4. Resultados. - Se propone un diseño para el paquete estructural el cual ayudará al confort y transitabilidad de los vehículos que pasen por la avenida Industrial, Lurín, Lima y está estructurado por $7.5 \mathrm{~cm}$ de carpeta asfáltica, $20 \mathrm{~cm}$ capa de base y $15 \mathrm{~cm}$ de subbase.

\begin{tabular}{cc}
\hline \multicolumn{2}{c}{ Características del pavimento } \\
\hline Carpeta & 3.0 \\
\hline Base $(\mathrm{cm})$ & 20.0 \\
\hline Sub Base & 15.0 \\
\hline
\end{tabular}

Tabla I. Características del pavimento flexible.

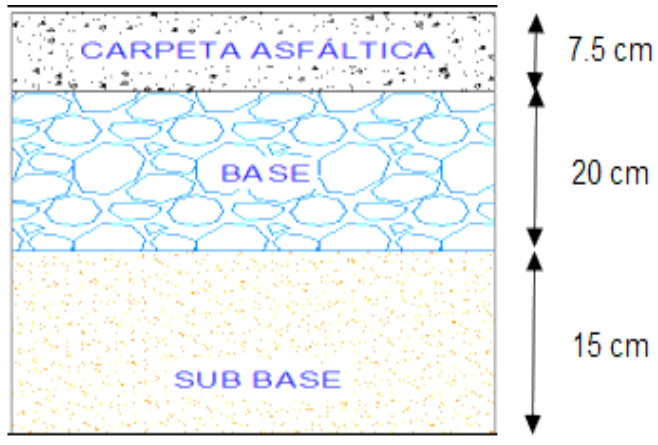

Figura I. Diseño estructural del pavimento. 
Según estudio de (CBR) realizado a la avenida Industrial, Lurín, Lima se realizaron 6 calicatas teniendo un promedio de CBR 32.50. La menor capacidad de carga del suelo encontrada en el estudio corresponde a las calicatas C-04, C-06 no obstante el resultado es considerado según su categoría como muy bueno.

\begin{tabular}{|c|c|c|c|c|c|c|}
\hline Calicata & Progresiva & Profundidad & SUCS & $\% \mathrm{CBR}$ & $\begin{array}{l}\text { CBR } \\
\text { PROM }\end{array}$ & MR \\
\hline $\mathrm{C}-01$ & $00+100.00$ & \multirow{6}{*}{$1.30-1.50$} & SP-SM & 38.00 & \multirow{6}{*}{32.5} & \multirow{6}{*}{$23,713.53$} \\
\hline $\mathrm{C}-02$ & $00+500.00$ & & SP-SM & 35.00 & & \\
\hline C-03 & $00+850.00$ & & SP & 38.00 & & \\
\hline $\mathrm{C}-04$ & $00+200.00$ & & SP-SM & 21.00 & & \\
\hline C-05 & $00+550.00$ & & SP-SM & 42.00 & & \\
\hline $\mathrm{C}-06$ & $00+900.00$ & & SP & 21.00 & & \\
\hline
\end{tabular}

Tabla II. Características del suelo para determinación de MR.

El estudio vehicular realizado durante 7 días, nos permitió clasificar cada tipo de vehículo y obtener un ESAL de 1.22 E+07. El diseño está proyectado para 20 años.

\begin{tabular}{lc}
\hline Días del año & 365 \\
\hline Factor direccional & 0.50 \\
\hline Factor de carril & 0.80 \\
\hline $\begin{array}{l}\text { Tasa anual de crecimiento } \\
\text { de transito }\end{array}$ & 3.00 \\
\hline $\begin{array}{l}\text { Número de años de periodo } \\
\text { de diseño }\end{array}$ & 20.00 \\
\hline ESAL & $1.22 \mathrm{E}+07$ \\
\hline
\end{tabular}

Tabla III. Determinación del ESAL.

5. Discusiones. - Se propone un diseño del pavimento flexible el cual servirá para mejorar la transitabilidad vehicular concordando con Montealegre y Betancourt (2019) que nos indica que la mejor opción para el cálculo del paquete estructural fue utilizar el método Aashto 93 por ser el método empírico más utilizado a nivel mundial y comparándolo con otros métodos.

Según estudio de capacidad de carga (CBR) realizado se obtuvo un promedio de CBR 32.50, la menor capacidad de carga del suelo encontrada en el estudio corresponde a las calicatas C-05, C06 no obstante el resultado es considerado según su categoría como muy bueno, concordando con Sánchez (2019) que indica que para el diseño final del pavimento se procede a tomar el promedio del CBR calculado en cada sector, difiere con Salamanca y Zuluaga (2014) nos indica que los estudios realizados a los suelos en el recorrido de $6.5 \mathrm{~km}$ de la vía dieron como resultado arenas limosas y limos arcillosos en toda el área de estudio.

6. Conclusiones. - Se propone un diseño del pavimento flexible, el cual servirá para mejorar la transitabilidad vehicular de la avenida Industrial, Lurín, Lima y está estructurado por $7.5 \mathrm{~cm}$ de carpeta asfáltica, $20 \mathrm{~cm}$ de base y $15 \mathrm{~cm}$ de subbase.

Según estudio de capacidad de carga (CBR) realizado a la avenida Industrial, Lurín, Lima se realizaron 6 calicatas teniendo un promedio de CBR 32.50. El estudio vehicular realizado durante 
7 días, para el diseño de pavimento flexible nos dio un ESAL de 1.22 E+07 realizado para un periodo de 20 años. Para el cálculo del diseño de pavimento flexible se ha utilizado el método Aashto 93 el cual mediante su ecuación de diseño nos permite calcular el número estructural.

7. Recomendaciones. - Los materiales para el asfalto, la base, subbase deberán cumplir las especificaciones técnicas establecidas por el MTC.

Para la preparación de la subrasante se tiene que eliminar toda materia orgánica.

La capa base granular de espesor $20 \mathrm{~cm}$, debe tener un CBR igual o mayor al 100\% de la MDS. Como el periodo de diseño está proyectado para 20 años, al concluir el periodo se recomienda realizar un nuevo estudio de tráfico vehicular. 


\section{Referencias.}

[1] Revista vial, La infraestructura vial es la columna vertebral de la economía mundial. [en línea]. Buenos Aires, mayo de 2010. [fecha de consulta: 12 de junio de 2021]. Disponible en https://revistavial.com/la-infraestructura-vial-es-la-columna-vertebral-de-la-economia-mundial/ [2] Ministerio de transporte y Comunicaciones, provias nacional. Estado de la red vial nacional. [en línea]. Lima, 2019. [fecha de consulta: 07 de julio de 2021]. Disponible en https://www.pvn.gob.pe/wp-content/uploads/2020/06/pvn-memoria-anual-2019.pdf

[3] M. Álamo [et al.]. Análisis general y diagnostico urbano de Lurín. Lima, 2015. [fecha de consulta: 25 de julio de 2021]. Disponible en https://fdocuments.in/document/diagnostico-urbanode-lurin.html

[4] Instituto nacional de estadística e informática, censo nacional, resultados definitivos. [en línea]. Lima, Octubre de 2017. [fecha de consulta: 30 de junio de 2021]. Disponible en https://www.inei.gob.pe/media/MenuRecursivo/publicaciones_digitales/Est/Lib1550/

[5] Municipalidad distrital de Lurin, gerencia de planeamiento estratégico informe de evaluación de resultados. [en línea]. Lima, abril de 2020. [fecha de consulta: 22 de junio de 2021]. disponible en https://munilurin.gob.pe/documentos/evaluaci\%c3\%93n\%20del\%20pei\%202020.pdf

[6] Ministerio de trabajo y promoción del empleo, desarrollo económico cono Sur. [en línea]. Lima, 2021. [fecha de consulta: 10 de julio de 2021]. Disponible en https://www.gob.pe/mtpe\#publicaciones

[7] Análisis del mercado industrial, parques industriales Lima centro, Lima norte, Lima este, Lima sur. Lima, 2020. [fecha de consulta: 20 de julio de 2021]. Disponible en https://www.cresperu.com/es/assets/uploads/mercado_pdf_Analisis_del_Mercado_Industrial_2T -2020.pdf

[8] C. Bermudez and Y. Ramos. Diseño estructural del pavimento flexible para el mejoramiento de la transitabilidad en la prolongación Av. Uno y la prolongación Sinchi Roca, La Libertad. Tesis (Ingeniero Civil). Trujillo: Universidad Privada Antenor Orrego, 2019. Disponible en https://repositorio.upao.edu.pe/bitstream/20.500.12759/5380/1/t_civ_carlos.bermudez_yuvickza.r amos_dise\%c3\%910.estructural_datos.pdf

[9] M. Ccasani y Y. Ferro. Evaluación y análisis de pavimentos en la ciudad de Abancay, para proponer una mejor alternativa estructural en el diseño de pavimentos. Tesis (Ingeniero Civil). Apurímac: Universidad Tecnológica de los Andes, 2017. Disponible en http://repositorio.utea.edu.pe/bitstream/handle/utea/107/Tesis-

Evaluaci\%c3\%b3n\%20y\%20an\%c3\%a1lisis\%20de\%20pavimentos\%20en\%20la\%20ciudad $\% 20$ de\%20Abancay\%201.pdf? sequence $=1 \&$ is Allowed $=y$

[10] Guía aashto 1993, Diseño de estructuras de pavimentos 1993. Instituto para el desarrollo de los pavimentos en el Perú. ed. Sociedad Americana de Oficiales Estatales de Carreteras y Transportes. Lima, 1997.

[11] E. Juárez y A. Rico. Mecánica de suelos tomo II. Teoría y aplicación de la mecánica de suelos. ed. Limusa S.A. Mexico.D.F, 2004. ISBN: 9789681800697

[12] C. Pereda y M. Montoya. Estudio y optimización de la red vial avenida América Sur tramo prolongación Cesar Vallejo - avenida Ricardo Palma, Trujillo. Tesis (Ingeniero Civil). Trujillo: Universidad Privada Antenor Orrego, 2018. Disponible en https://repositorio.upao.edu.pe/bitstream/20.500.12759/4178/1/rep_ing.civil_christopher.pereda_ mario.montoya_estudio.optimizacion.red.vial.avenida.america.sur.tramo.prolongaci\%c3\%92n.ce sar.vallejo.avenida.ricardo.palma.trujillo.pdf

[13] Manual de carreteras. Sección suelos y pavimentos. [en línea]. Lima, abril de 2014. [fecha de consulta: 20 de junio de 2021]. Disponible en https://portal.mtc.gob.pe/transportes/caminos/normas_carreteras/documentos/manuales/manuales $\% 20 \mathrm{de} \% 20$ carreteras\%202019/mc-05- 
[14] L. Escobar and J. Huincho. Diseño de pavimento flexible, bajo influencia de parámetros de diseño debido al deterioro del pavimento de Santa Rosa - Sachapite. Tesis (Ingeniero Civil). Huancavelica: Universidad Nacional de Huancavelica, 2017. Disponible en file://C:/Users/guirl/Downloads/TP\%20-\%20UNH\%20CIVIL.\%200085\%20(3).pdf

[15] H. Rondon and F. Reyes. Pavimento Materiales, construcción y diseño. ed. Ecoe ediciones S.A. Bogota, 2015. ISBN: 9789587711769

[16] J. Menéndez. Ingeniería y diseño de pavimentos. ed. Instituto de la construcción y gerencia. Lima, 2017. ISBN: 9786124280153

[17] Z. Vargas. Investigación aplicada, una forma de conocer las realidades con evidencia científica. San Pedro Montes de Oca - Costa Rica. Revista científica, 2009. ISSN: 0379-7082

[18] G Mousalli. Métodos y diseños de investigación cuantitativa. Mérida, [en línea]. Venezuela, 2015. [fecha de consulta: 30 de junio de 2021]. Disponible en file:///C:/Users/guirl/Downloads/Mtodosydiseosdelainvestigacincuantitativa\%20(1).pdf

[19] R. Hernández, C. Fernández and M. Baptista. Metodología de la investigación. 6ta. ed. McGraw-Hill / interamericana editores S.A. de c.v. México D.F, 2014. ISBN: 9781456223960

[20] C. Bernal. Metodología de la investigación administración, economía, humanidades y ciencias. 3ra. ed. Pearson educación S.A. Colombia, 2010. ISBN: 978-958-699-128-5 